\title{
Cortical herniation through compressive subdural membrane in an infant with a history of a large bihemispheric subdural hematoma and subdural-peritoneal shunt: case report
}

\author{
Aleka Scoco, BA, ${ }^{1}$ E. Emily Bennett, MD, MS, ${ }^{2}$ and Violette Recinos, MD ${ }^{2}$ \\ ${ }^{1}$ Case Western Reserve University School of Medicine; and ${ }^{2}$ Department of Neurological Surgery, Neurological Institute, \\ Cleveland Clinic, Cleveland, Ohio \\ Cortical herniation through subdural membrane formation is a rare complication of chronic subdural fluid collections and \\ may occur following subdural shunting. The authors present a unique case of progressive cortical herniation through a \\ compressive subdural membrane that occurred concomitant with a functioning subdural-peritoneal shunt. \\ https://thejns.org/doi/abs/10.3171/2016.8.PEDS16108
}

KEY WORDS armored brain; subdural hemorrhage; cortical herniation; pediatric; hydrocephalus

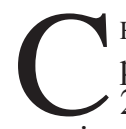

HRONIC subdural fluid collections in the pediatric population largely occur in infants younger than 2 years, often secondary to trauma, and can communicate when bilateral. ${ }^{1-3,21,26}$ Common complications of such collections include brain atrophy, cephalic enlargement, seizure disorder, intracranial hypertension, visual loss, and developmental delay. ${ }^{1}$ When clinically significant, drainage of the subdural collections via bur hole or craniotomy is indicated. When drainage alone is insufficient, subdural-peritoneal shunting is a safe and effective treatment and has been shown to result in complete clinical and radiographic resolution of subdural fluid collections in a majority of cases. $7,8,11,12,18,20$

Subdural neomembrane formation is a rare complication that may result from chronic subdural fluid collections. If this subdural neomembrane forms, cortical herniation may also be present. A total of 4 pediatric cases have been reported in the literature, with patients ranging in age from 11 to 14 months (Table 1). ${ }^{1,5,15,16}$ We present a unique case of cortical herniation through a compressive subdural neomembrane that occurred concomitant with a functioning subdural-peritoneal shunt.

\section{Case Report}

History and Examination

This patient initially presented to his primary care phy- sician with increasing head circumference and a bulging anterior fontanelle when he was 3 months old. He was admitted to the hospital and was found to have a large, subacute to chronic, bilateral subdural hemorrhage (SDH) measuring $3.6 \mathrm{~cm}$ in greatest transverse diameter and a linear, nondepressed right parietal skull fracture on head CT scanning with an unclear history of possible minor trauma. The etiology was unclear, but there was suspicion of nonaccidental trauma (NAT), given the patient's imaging findings and bilateral retinal hemorrhages found on ophthalmological examination.

The patient subsequently underwent uncomplicated placement of bilateral parietal bur holes for SDH drainage with bilateral subdural drain placement. Postoperatively, he remained at baseline without neurological deficit, and the anterior fontanelle was soft and sunken. The patient was weaned from the subdural drains, and on postoperative Day 3, the drains were removed after imaging did not reveal any significant reaccumulation. The patient was discharged and monitored closely as an outpatient. Over the next 2 weeks, he again developed increasing head circumference and progressive fullness of his anterior fontanelle. Serial head ultrasound studies confirmed significant fluid reaccumulation. He underwent left-sided subduralperitoneal shunt placement (Codman Hakim Programmable Valve System) set at $30 \mathrm{~cm} \mathrm{H}_{2} \mathrm{O}$, the lowest resistance

ABBREVIATIONS NAT = nonaccidental head trauma; $\mathrm{SDH}=$ subdural hematoma . 


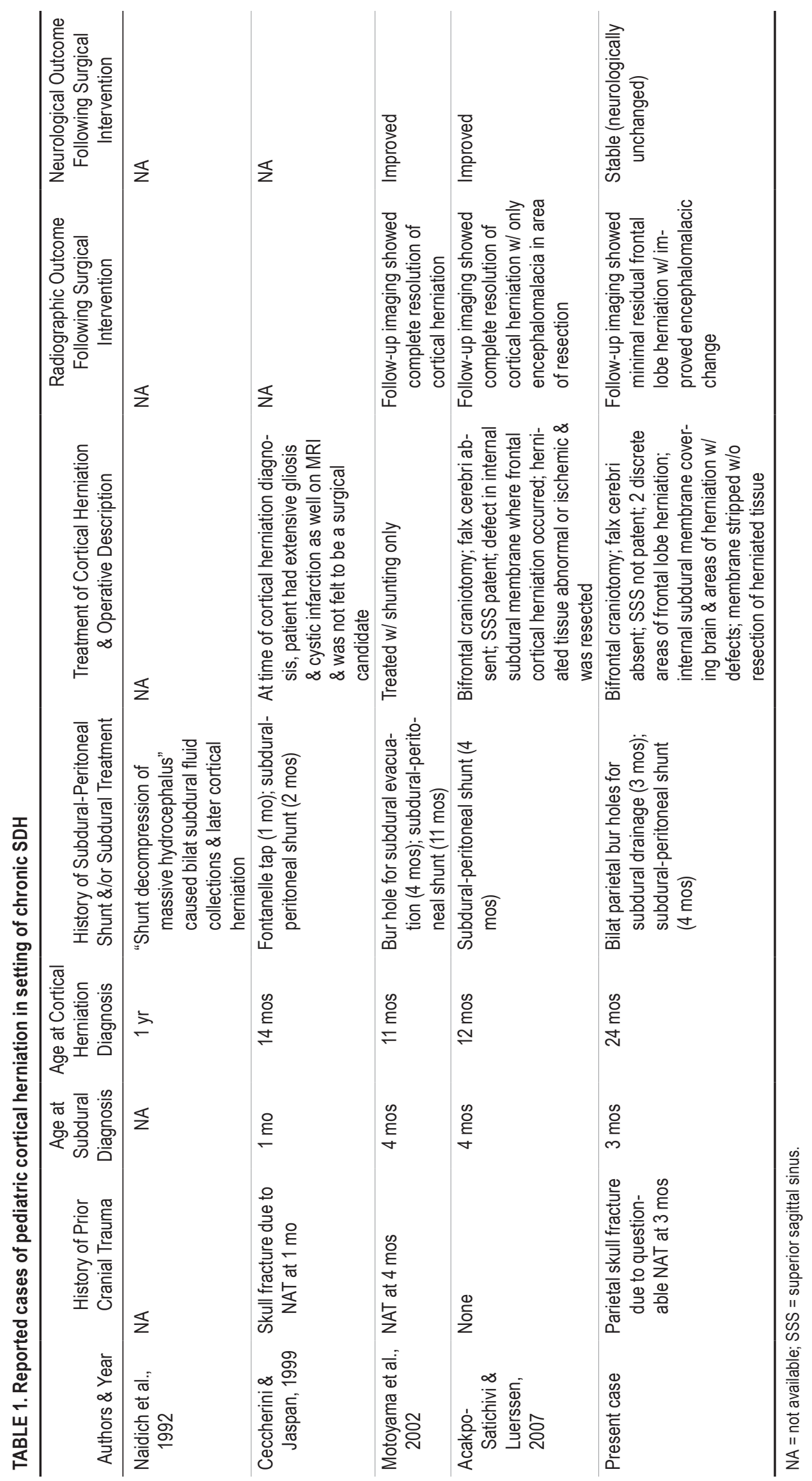


setting. The patient had an uncomplicated postoperative course with stabilization of his head circumference and return of his anterior fontanelle to flat or sunken on serial neurological examinations.

The patient was monitored closely over the following year with head ultrasound studies, which showed improving but persistently large subdural hygromas. Despite this, he continued to have a stable head circumference and small sunken fontanelle; he was developing well and remained asymptomatic. At 18 months of age, the patient underwent an MRI study of his brain for routine followup, which showed persistent subdural hygromas. Additionally, there appeared to be continued mass effect on the underlying brain parenchyma as well. At the time of the MRI study, the family reported episodic nausea and vomiting that had just started 1 week earlier. Physical examination revealed a depressed and nonrefilling valve reservoir, and ophthalmological examination revealed papilledema. He underwent shunt exploration and revision for an obstructed proximal catheter and valve, and also required distal catheter lengthening. The patient returned to his baseline condition immediately after the procedure.

Six months later, the patient underwent another routine follow-up MRI study, which showed persistent bilateral subdural hygromas and continued apparent mass effect on the cortex. Imaging revealed more clearly a compressive neomembrane constricting the underlying parenchyma and limiting brain expansion. Multiple areas of frontal lobe tissue herniated through defects in this compressive neomembrane. The herniated tissue was cystic with encephalomalacia (Fig. 1). Retrospective review of the patient's prior MRI study showed evidence of frontal lobe herniation as well, but the neomembrane was more clearly identified and the degree of brain herniation significantly worsened in the 6-month interval (Fig. 2). Despite these imaging findings, the patient continued to develop well without any signs or symptoms of elevated intracranial
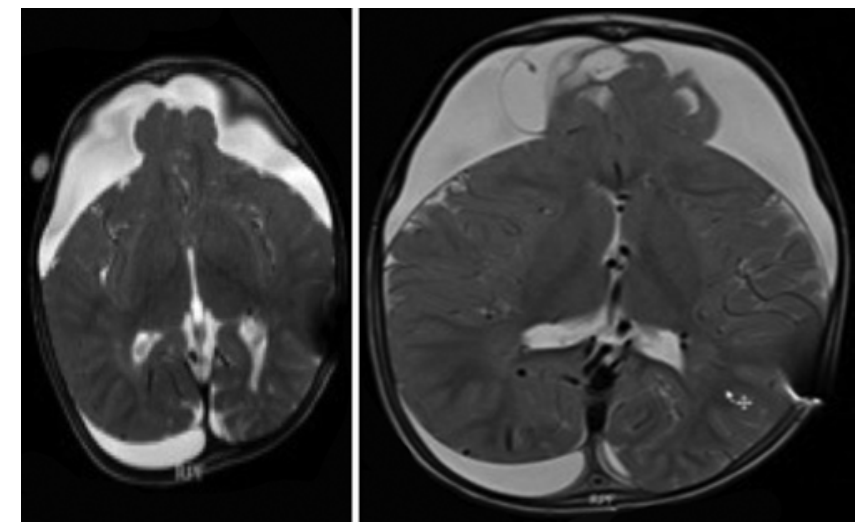

FIG. 2. Direct comparison of axial T2-weighted MR images obtained when the patient was 18 months old (left) and 6 months later (right), showing more clearly the subdural neomembrane and worsening frontal lobe herniation.

pressure or focal neurological defects. His shunt reservoir depressed and refilled easily, and there was no papilledema. The patient's head circumference had remained stable after the shunt was implanted. There were no findings to suggest shunt malfunction. The MRI study also revealed apparent attenuation of the sagittal and transverse sinuses. Therefore, MR angiography and MR venography were performed 1 month later and showed a diminutive appearance of the superior sagittal sinus throughout its course with multiple collateral venous channels draining into the deep venous systems. Note was made of minimal draining veins on the cortical surface. Additionally, MR angiography revealed that the distal anterior cerebral arteries were also herniated into the frontal lobe defects.

The imaging findings showing progressive cortical herniation and persistent parenchymal compression raised concern that further herniation might result in detrimental neurological and developmental sequelae. Options for
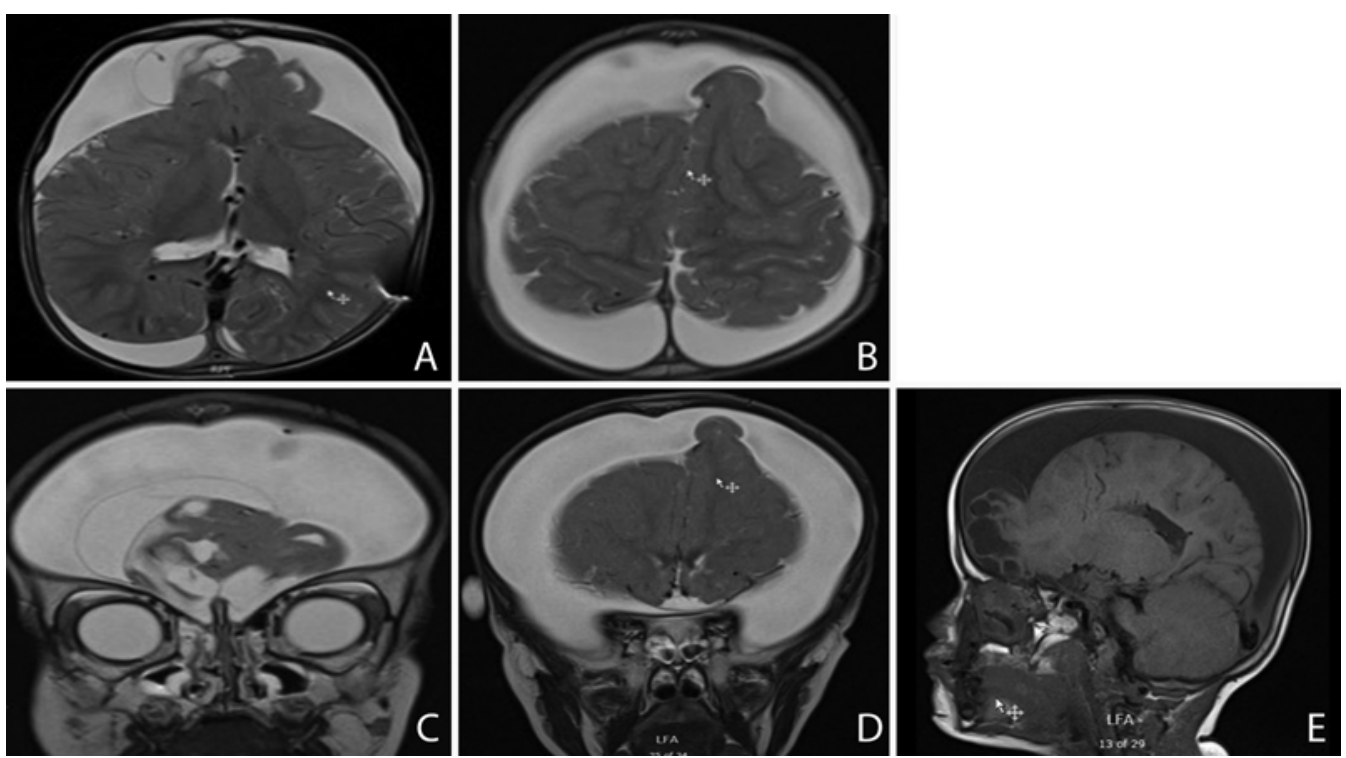

FIG. 1. Axial (A and B) and coronal (C and D) T2-weighted MR images and sagittal T1-weighted image (E) showing bilateral chronic subdural collections with 2 areas of frontal lobe herniation through a compressive neomembrane with cystic/encephalomalacic changes. 
management were discussed at length with the family, including continued monitoring with serial images versus surgical intervention. Despite the patient's clinical stability, the progressive imaging findings prompted us to offer a bilateral craniotomy to strip the compressive membranes and release the herniated brain tissue. We also considered the possibility that the shunt might not have been functioning properly, which may have contributed to the ongoing pathology. However, the patient did not demonstrate any signs or symptoms of shunt malfunction like he had in the past. Plans were made to explore the shunt first at the beginning of the procedure to ensure that the shunt was functioning properly prior to proceeding with the more invasive craniotomy and membrane stripping. The patient's family agreed to proceed.

\section{Operation}

The patient was given a perioperative dose of levetiracetam for seizure prophylaxis. A bicoronal incision was planned, incorporating the left subdural shunt incision. Starting at the shunt site, the shunt was exposed and disconnected. All components were found to be functioning properly. A large bifrontal craniotomy was then performed. The dura was boggy, and the superior sagittal sinus was noted to be completely atretic anteriorly. After opening the dura and draining the large subdural hygromas, a whitish, iridescent compressive membrane was visualized covering the entire brain surface, with the exception of small defects over the areas of cortical herniation (Fig. 3). There were 2 focal regions of frontal lobe herniation (Fig. 3) with a band of membrane around the "neck" of the herniated tissue. This cortical tissue was grossly abnormal but was not resected due to concern for functional tissue and vasculature traveling through the tissue. The lack of cortical surface veins was also noted. There were several small adhesions extending from the cortex to the dura that appeared to be remnants of prior small veins and were now completely atretic. Despite the neomembrane's adherence to the underlying arachnoid, the membrane quite easily separated from the underlying brain; the lack of cortical surface veins allowed for a virtually bloodless dissection of the membrane. In a slow but deliberate fashion, the membrane was stripped successfully from the exposed cortical surface and from around the herniation sites (Fig. 4).

The proximal subdural shunt catheter was removed after opening the dura and was replaced at the end of the procedure. Because of the large epidural space created after the drainage of the subdural hygroma, the valve setting was increased to $60 \mathrm{~cm} \mathrm{H}_{2} \mathrm{O}$ to prevent epidural hematoma formation in the postoperative period.

Histopathological analysis of the subdural membrane showed a benign, fibrous walled cyst with focal macrophages.

\section{Postoperative Course}

After surgery, the patient remained at neurological baseline, without any new neurological deficit. He was discharged home on postoperative Day 4. He continued receiving levetiracetam through the postoperative period and was weaned off of the medication over 1 month.

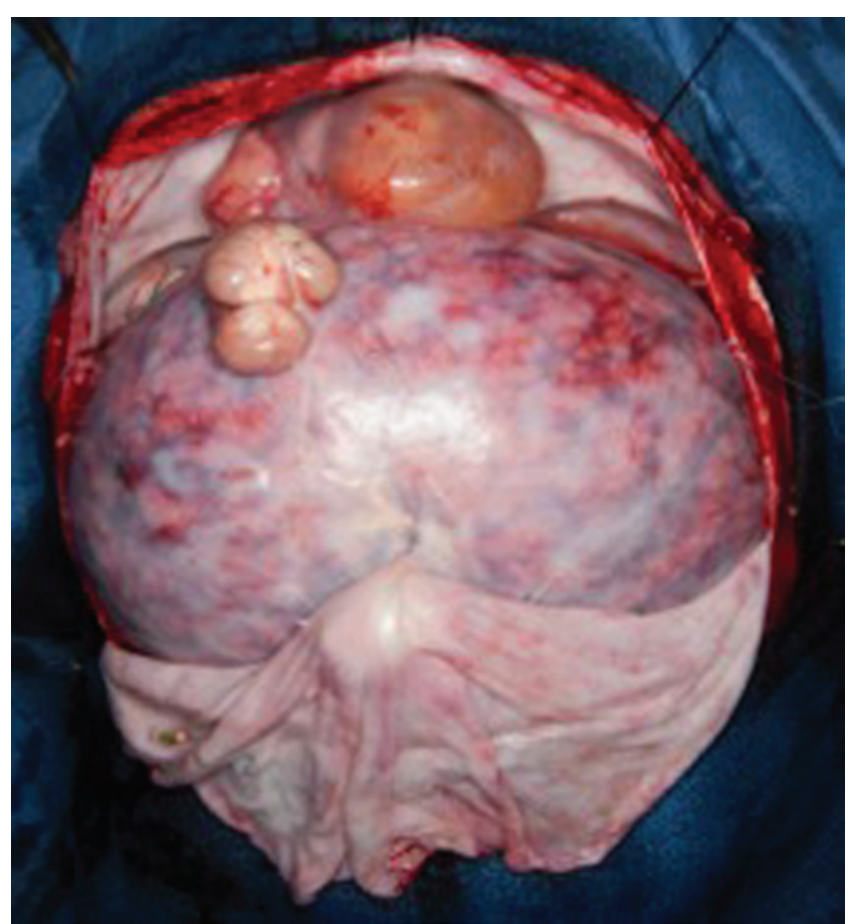

FIG. 3. Intraoperative photograph. After dural opening there is a clear separation of the subdural neomembrane from the dura. Figure is available in color online only.

The patient experienced a single seizure 2 months postoperatively, which was successfully treated by reinstating levetiracetam with no further seizure activity to date. At 3 months postoperatively, the patient returned to the clinic for routine follow-up MRI, which showed decreasing hygromas and marked resolution of the frontal lobe herniation with improved encephalomalacic change. The valve could not be reprogrammed following MRI (it appeared to be set at $30 \mathrm{~cm} \mathrm{H}_{2} \mathrm{O}$ ), and the skin overlying the valve was noted to have chronic wound irritation. Because the patient clinically appeared well, he was monitored, but over the next few days he became increasingly irritable with vomiting. Follow-up examination revealed that the shunt reservoir was depressed and not refilling. Therefore, he underwent an uncomplicated shunt and wound revision with replacement of an occluded proximal catheter and valve and has since been doing well. His latest MRI study at 7 months after his surgery shows further resolution of
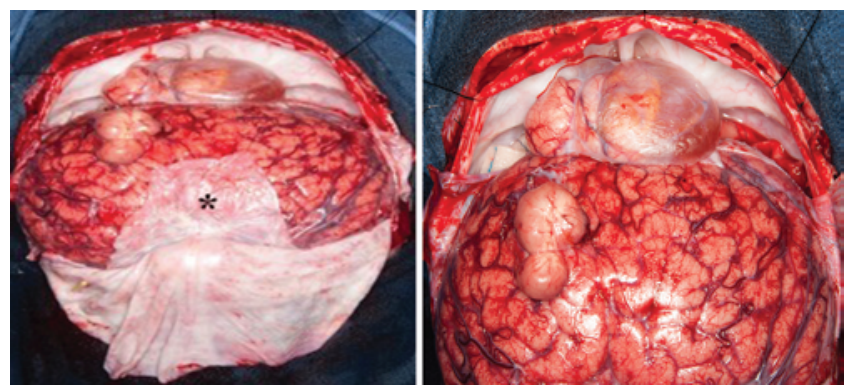

FIG. 4. Intraoperative photographs. Two views of the cortical brain surface after subdural neomembrane stripping. The asterisk denotes the subdural neomembrane. Figure is available in color online only. 

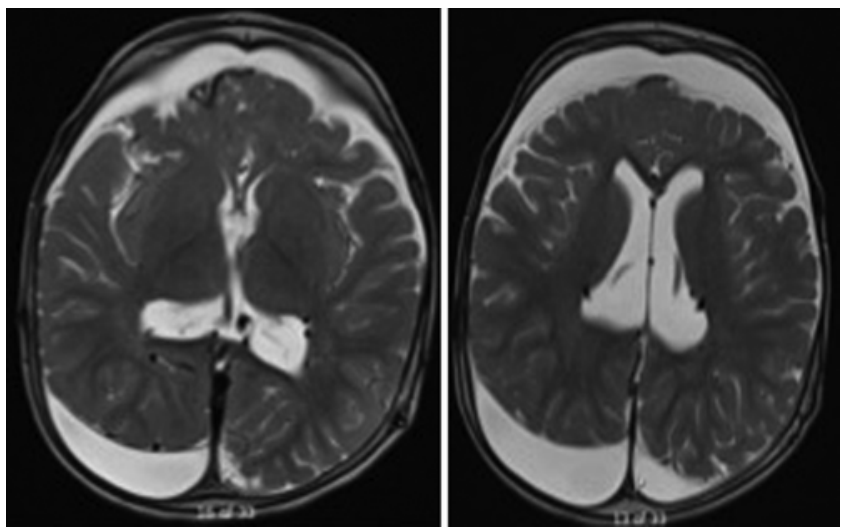

FIG. 5. Axial T2-weighted MR images of the brain obtained 7 months postoperatively showing minimal residual frontal lobe herniation with improved encephalomalacic change and a decrease in the size of the bilateral subdural fluid collections.

the previous areas of cortical herniation and decreased bilateral hygromas (Fig. 5).

\section{Discussion}

Inner subdural neomembrane formation has been described in the literature as a potential complication of chronic SDH; however, most of the literature focuses on the evolution of an "armored brain" or calcified subdural membrane. . $9,22,25,27,28,30$ Pediatric patients also can exhibit subdural neomembrane formation that is not as heavily calcified but is highly restrictive in the setting of chronic $\mathrm{SDH} .1,5,15$ The membrane is generally divided into 2 layers, with the thicker, outer neomembrane forming between the dura and the expanding clot and a thinner, inner membrane just superficial to the arachnoid layer; both develop from granulation tissue formation, with increasing vascularity over time. ${ }^{6,10}$ Visualization of 2 distinct neomembranes is often impossible, as in the present case..$^{10}$

Cortical herniation with an associated restrictive thickened neomembrane is an extremely rare complication of chronic SDH. The first reported case was termed "internal encephalocele" in a 1-year-old girl in 1992, and described an initial event, such as trauma or surgery, creating new intraaxial membrane partitions with potential opportunities for brain herniation. ${ }^{16}$

Ceccherini and Jaspan described a case similar to ours of a 14-month-old child and posited that cerebral pulsations, already known to play a role in growing skull fractures and leptomeningeal cyst formation, could have caused extrusion of tissue through the defect. ${ }^{5,17,29}$ Yet, similar to our case, the site of cortical herniation was far from the site of the original skull fracture and completely healed when herniation was discovered. ${ }^{5}$ The authors suggested that focal tethering of the brain to the subdural neomembrane could contribute to herniation. ${ }^{5}$ Another report, which described resolution of symptoms after placement of a subdural-peritoneal shunt, proposed that the rapidity of brain growth and flexibility afforded by lack of myelination as possible etiological mechanisms for pediatric cases of intracranial encephalocele. ${ }^{15}$

Another interesting aspect of this case was the atretic superior sinus and formation of deep collateral venous channels, although, to our knowledge, in no prior case of subdural membrane with cortical herniation was a redistribution of cerebral venous drainage observed. Although the etiology of our patient's SDHs was suspected to be due to NAT, this was never proven. This could raise speculation regarding what role this venous anomaly had in this patient's pathology. There have been case reports of children with benign enlargement of the subarachnoid space developing spontaneous subdural hemorrhages in the absence of severe trauma. ${ }^{13,25}$ Perhaps a preexisting venous anomaly could contribute to the development of benign enlargement of the subarachnoid space and predispose the patient to the SDH. Furthermore, the absence of arachnoid granulations and superficial drainage may also contribute to the lack of physiological CSF absorption and continued dependence on the subdural-peritoneal shunt for ongoing CSF drainage.

Similar to the patient reported on by Acakpo-Satchivi and Luerssen, our patient developed cortical herniation after several months of a functioning subdural-peritoneal shunt. ${ }^{1}$ This suggests that shunting alone may not be a successful treatment, contrary to the discussion by Motoyama et al. ${ }^{1,15}$ Shunting was performed in all of these cases of cortical herniation in patients ranging from 11 to 14 months; other authors have suggested that shunting is necessary to prevent neurological decline, which was further supported by the present case in a 2-year-old child. ${ }^{1,5,15,16}$

It is still unclear why areas of cortical herniation develop in the setting of chronic pediatric SDH. Subdural membrane excision has been proven to be unnecessary for resolution of subdural effusions and can lead to cortical irritation, bleeding, and seizures postoperatively. ${ }^{9,14,24}$ However, in select cases like the present one, the presence of persistent intracranial hypertension despite appropriate treatment necessitates membrane removal for resolution of symptoms and to prevent future decline., ${ }^{4,14}$ This operation was highly unusual, and we would not recommend it as a standard initial approach for children with chronic SDHs. The membrane in our patient was directly linked to underlying parenchymal compression as evidenced by postoperative cerebral expansion following membrane stripping.

On follow-up imaging, the subdural fluid collections decreased in size and the areas of cortical herniation had improved. This further suggests that the subdural membrane was responsible for underlying compression instead of the subdural fluid collections. Only one other report has described cortical herniation in the setting of a functional subdural-peritoneal shunt. ${ }^{1}$ In that report, the authors suggested that the presence of the functioning shunt may have accelerated the herniation, and they also found improvement in the areas of cortical herniation following membrane stripping. In our case, we too hypothesized that a low shunt setting might have also contributed to the progressive herniation of cortical tissue through the membrane by creating a pressure differential between the space above and below the membrane. We considered adjusting the shunt to a higher setting as an initial management. However, the large, persistent subdural space and concern for poor physiological CSF absorption led us to favor membrane stripping as the best long-term solution to the problem. 
The treatment of chronic SDHs in infancy remains challenging. Our case is a unique example of a compressive membrane resulting in cortical herniation following subdural hemorrhage. Our approach therefore is not typical and is not recommended as a standard of care. Furthermore, this case illustrates that in infants who suffer head trauma and require subdural shunt placement, serial follow-up is recommended. Additional cases of chronic SDHs in infants and their subsequent treatment and follow-up will be useful in providing more insight into the development of such complications.

\section{Conclusions}

This case illustrates a rare complication of chronic $\mathrm{SDH}$ in the setting of a functional subdural-peritoneal shunt. Radiographic evidence of a compressive subdural neomembrane, when concomitant with signs of intracranial hypertension and cortical herniation, should serve as an indication for surgical intervention to prevent brain strangulation and potential neurological complications.

\section{References}

1. Acakpo-Satchivi L, Luerssen TG: Brain herniation through an internal subdural membrane: a rare complication seen with chronic subdural hematomas in children. Case report. J Neurosurg 107 (6 Suppl):485-488, 2007

2. Aoki N: Chronic subdural hematoma in infancy. Clinical analysis of 30 cases in the CT era. J Neurosurg 73:201-205, 1990

3. Aoki N, Mizutani H, Masuzawa H: Unilateral subduralperitoneal shunting for bilateral chronic subdural hematomas in infancy. Report of three cases. J Neurosurg 63:134-137, 1985

4. Caldarelli M, Di Rocco C, Romani R: Surgical treatment of chronic subdural hygromas in infants and children. Acta Neurochir (Wien) 144:581-588, 2002

5. Ceccherini AF, Jaspan T: Cerebral herniation through a subdural membrane defect following non-accidental injury. Clin Radiol 54:550-552, 1999

6. Collins WF, Pucci GL: Peritoneal drainage of subdural hematomas in infants. J Pediatr 58:482-485, 1961

7. Erşahin Y, Mutluer S: A method for continuous external drainage in the management of infantile subdural collections. Childs Nerv Syst 11:418-420, 1995

8. Gaskill SJ, Oakes WJ, Marlin AE: Continuous external drainage in the treatment of subdural hematomas of infancy. Pediatr Neurosurg 17:121-123, 1991-1992

9. Herzberger E, Rotem Y, Braham J: Remarks on thirty-three cases of subdural effusion in infancy. Arch Dis Child 31:4450,1956

10. Ingraham FD, Matson DD: Subdural hematoma in infancy. $\mathbf{J}$ Pediatr 24:1-37, 1944

11. Korinth MC, Lippitz B, Mayfrank L, Gilsbach JM: Subduralatrial and subdural-peritoneal shunting in infants with chronic subdural fluid collections. J Pediatr Surg 35:1339-1343, 2000

12. Litofsky NS, Raffel C, McComb JG: Management of symptomatic chronic extra-axial fluid collections in pediatric patients. Neurosurgery 31:445-450, 1992

13. Mattei TA, Sambhara D, Bond BJ, Lin J: Clinical outcomes of temporary shunting for infants with cerebral pseudomeningocele. Childs Nerv Syst 30:283-291, 2014
14. McLaurin RL, Isaacs E, Lewis HP: Results of nonoperative treatment in 15 cases of infantile subdural hematoma. J Neurosurg 34:753-759, 1971

15. Motoyama Y, Isaka F, Nabeshima S: Internal intracranial encephalocele reduced by a subdural-peritoneal shunt. Case illustration. J Neurosurg 96:966, 2002

16. Naidich TP, Altman NR, Braffman BH, McLone DG, Zimmerman RA: Cephaloceles and related malformations. AJNR Am J Neuroradiol 13:655-690, 1992

17. Ommaya AK, Grubb RL Jr, Naumann RA: Coup and contrecoup injury: observations on the mechanics of visible brain injuries in the rhesus monkey. J Neurosurg 35:503-516, 1971

18. Parent AD: Pediatric chronic subdural hematoma: a retrospective comparative analysis. Pediatr Neurosurg 18:266271,1992

19. Ramachandran R, Hegde T: Chronic subdural hematomascauses of morbidity and mortality. Surg Neurol 67:367-373, 2007

20. Ransohoff J: Chronic subdural hematoma treated by subdural-pleural shunt. Pediatrics 20:561-564, 1957

21. Sauter KL: Percutaneous subdural tapping and subdural peritoneal drainage for the treatment of subdural hematoma. Neurosurg Clin N Am 11:519-524, 2000

22. Schachenmayr W, Friede RL: The origin of subdural neomembranes. I. Fine structure of the dura-arachnoid interface in man. Am J Pathol 92:53-68, 1978

23. Shimoji T, Sato K, Ishii S: [A pathogenesis of chronic subdural hematoma; it's relationship to subdural membrane.] No Shinkei Geka 20:131-137, 1992 (Jpn)

24. Shulman K, Ransohoff J: Subdural hematoma in children: the fate of children with retained membranes. J Neurosurg 18:175-181, 1961

25. Suara RO, Trouth AJ, Collins M: Benign subarachnoid space enlargement of infancy. J Natl Med Assoc 93:70-73, 2001

26. Swift DM, McBride L: Chronic subdural hematoma in children. Neurosurg Clin N Am 11:439-446, 2000

27. Taha MM: Armored brain in patients with hydrocephalus after shunt surgery; review of the literatures. Turk Neurosurg 22:407-410, 2012

28. Tanaka Y, Ohno K: Chronic subdural hematoma-an up-todate concept. J Med Dent Sci 60:55-61, 2013

29. Taveras JM, Ransohoff J: Leptomeningeal cysts of the brain following trauma with erosion of the skull; a study of seven cases treated by surgery. J Neurosurg 10:233-241, 1953

30. Yamashima T, Yamamoto S: The origin of inner membranes in chronic subdural hematomas. Acta Neuropathol 67:219225,1985

\section{Disclosures}

The authors report no conflict of interest concerning the materials or methods used in this study or the findings specified in this paper.

\section{Author Contributions}

Conception and design: Recinos, Bennett. Acquisition of data: Scoco, Bennett. Analysis and interpretation of data: all authors. Drafting the article: Scoco, Bennett. Critically revising the article: all authors. Reviewed submitted version of manuscript: all authors. Approved the final version of the manuscript on behalf of all authors: Recinos.

\section{Correspondence}

Violette Recinos, Cleveland Clinic, Neurological Institute, 9500 Euclid Ave. S60, Cleveland, OH 44195. email: recinov@ccf.org. 